\title{
IMPACT OF CONSISTENT FibRE PROPERTIES ON OPTIMIZATION OF FABRIC BARRÉ
}

\author{
Md. Sanuwar Rashid \\ Department of Textile Engineering \\ Daffodil International University \\ E-mail: sanuwar_69@yahoo.com
}

\begin{abstract}
Cotton is a natural fibre. It's growth depends upon the seasonal weather, climate condition and affection of paste and insects. So the properties of cotton fibres vary not only from lot to lot but also in same lot. The properties such as Micronaire, color grade, maturity measure the ability of dye absorbency of fibre. So if there is high variation of these properties in cotton then a significant variation of dye absorbency in fabric is observed. So the fabric, made form cotton yarn having inconsistent properties, generate complain of barré marks known as patta to our local people. To eliminate or minimize the problem of barré we must have to ensure the uniform distribution of cotton properties throughout the yarn manufacturing stages. And Bale Management is the procedure of determining mix laydowns by maintaining an appropriate combination of cotton properties with consistency through several mixes. Short and long term variation of fibre properties with in and between mixes is responsible for fabric barré which is possible to avoid by bale management.
\end{abstract}

Keywords: Barré, micronaire, color grade, immaturity, mixing, laydown.

\section{Introduction}

In textile production, one of the most common and often perplexing quality control problems is barré - repetitive yarn direction streaks. Approximately $70 \%$ of barré problem in finished fabric originate from the presence of fibre in yarn having a wide range of various properties, as like Micronaire, yellowness(+b), and Reflectance(Rd), immaturity(IFC). Another $10 \%$ problem is contributed by the fluctuation of processing parameter of yarn manufacturing stage [1]. So yarn manufacturer often faces complain from further processing zone of barré marks where as barré problem is not only caused by yarn manufacturing zone, the related causes may implies in tension variation of circular knitting machine or not uniform dye fixation in fabric surface. The return of goods (Yarn) to the producer with a replacement claim is usually quite costly. So it is very necessary to take to take steps in this regard.

\section{Definition of Barré}

The noun "barré" is defined by ASTM as an unintentional, repetitive visual pattern of continuous bars and stripes usually parallel to the filling of woven fabric or to the courses of circular knit fabric. In a warp knit fabric, barré normally runs in the length direction, following the direction of yarn flow. Barré can be caused by physical or optical or dye differences in the yarn, geometric difference in the fabric structure or by any combination of these difference.

According to The Textile Institute "fabric barré comprises of unwanted stripes in the woven fabrics in the direction of the weft. This fault also appears in weft knitted fabrics Usually on a multifeeder machine and consists of light or dark course-wise stripes arising from differences in luster, dye-affinity (unlevel dyeing) in the yarn, yarn spacing or loop length, yarn linear density or defecting plating” [2].

\section{Causes of Barré}

The varied and diverse causes of barré can generally be summed up in one wordINCONSISTENCY. An inconsistency which leads to barré can originate from the spinning mills in one or more of the following categories

- $\quad$ raw material (Fibre),

- yarn manufacturing process,

- lot wise yarn supply

With these three categories, factors which may cause or contribute to barré were introduce in [3] and are listed below:

\subsection{Raw Material Fibre}

Raw material is received in warehouse according to lot wise. Each lot of cotton owns some specific quality characteristics which 
do not match with other lot. Different origin of cotton possesses different Micronaire value and different color grade. So we have to make efficient blending (bale management) on the basis of permutation and combination of different lot to achieve average and consistent quality parameter, because fabric barré can be generated by the following:

1. Failure to control fibre diameter (micronaire) from laydown to laydown \& too high C.V. of micronaire in the laydown

2. Failure to control the range of fibre color in each laydown $(\mathrm{Rd},+\mathrm{b})$

Seasonal influence during cotton growth, including the influence of weather and climate or infestation by insects or fungi may also be responsible for barré effect. We also ensure the satisfactory level and range of maturity ratio or immature fibre content (IFC) for each laydown to get a constant dye fixation ability of fabric.

\subsection{Yarn manufacturing process}

1. Variation in carding; Different amount of non lint content removal from card to card.

2. Poor blending of fibre in opening through finisher drawing.

3. Running different types of spindle tapes on ring spinning frame.

4. All cots running in a given set of ring frame producing yarn for the same end use should exactly the same.

\subsection{Lot wise yarn supply}

1. Mixing of yarns of different count \& spinning system \& different blend level.

2. Mixing yarn of different twist level or twist direction.

3. Mixing yarn with different degrees of hairiness.

4. Excessive back winding or abrasion during this process.

5. If yarns are conditioned then each lot must be uniformly conditioned.

\section{Properties Related with Barré}

The contractual parameter, by which a lot of cotton consignment is bought, are length in inch, strength in gm/tex, Micronaire and color grade. Among these, length and strength are responsible for (i) hairiness level (ii) the strength of yarn (iii) efficiency of various stage of spinning mills. But they are not directly related with the dye take up\% or dye fixation of yarn or fabric on further processing. So we have to emphasis on other two properties, eg. Micronaire and Color grade due to their direct affection on dye absorbency and these were introduced by [4]

4.1 Contribution of MIC in fabric Barré Micronaire is a measure of fibre fineness and maturity. An air flow instrument is used to measure the air permeability of a constant mass of cotton fibres compressed to a fixed volume. Figure 1 can be used as a guide in interpreting micronaire measurements.

\begin{tabular}{|l|l|l|l|l|}
\hline B.4 \& & $3.5-$ & $\begin{array}{c}3.7-4.2 \\
\text { Premium } \\
\text { range }\end{array}$ & $\begin{array}{c}4.3- \\
4.9\end{array}$ & $\begin{array}{l}5.0 \& \\
\text { Up }\end{array}$ \\
\cline { 2 - 3 } & \multicolumn{3}{|c|}{ Base Range } \\
Discount Range \\
\hline
\end{tabular}

Fig. 1 Interpreting micronaire measurements

Micronaire measurement can be influenced during the growing period by environmental conditions such as moisture, temperature, sunlight, plant nutrients, and extremes in plant or boll population. Fibre fineness affects processing performance and the quality of the end product in several ways. In the opening, cleaning and carding processes, low micronaire or fine fibre, cotton require slower processing speeds to prevent damage of the fibres. Yarn made from the finer fibre result in more fibres per cross section, which in turn produce stronger yarn. Dye absorbency and retention varies with the maturity of the fibres. The greater the maturity, the better the absorbency and retention.

4.2 Contribution of Color in Fabric Barré The color of cotton is determined by the degree of reflectance $(\mathrm{Rd})$ and yellowness (+b). Reflectance indicates how bright or dull a sample is, and yellowness indicates the degree of pigmentation. A three digit color code is used. The color code is determined by locating the point at which the Rd and $+b$ values intersect on the Nickerson-Hunter cotton colorimeter diagram for upland cotton. The color of cotton fibres can be affected by rainfall, freezes, insects and fungi, and by staining through contact with soil, grass, or the cotton plant's leaf. Color can also be 
affected by excessive moisture and temperature levels while cotton is being stored, both before and after ginning. As the color of cotton deteriorates due to environmental condition, the probability for reduced processing efficiency is increased. Color deterioration mainly affects the ability of fibres to absorb and hold dyes and finishes. A color chart is taken in here from [5] and shown in Fig. 2.

FOR AMERICAN UPLAND COTTON

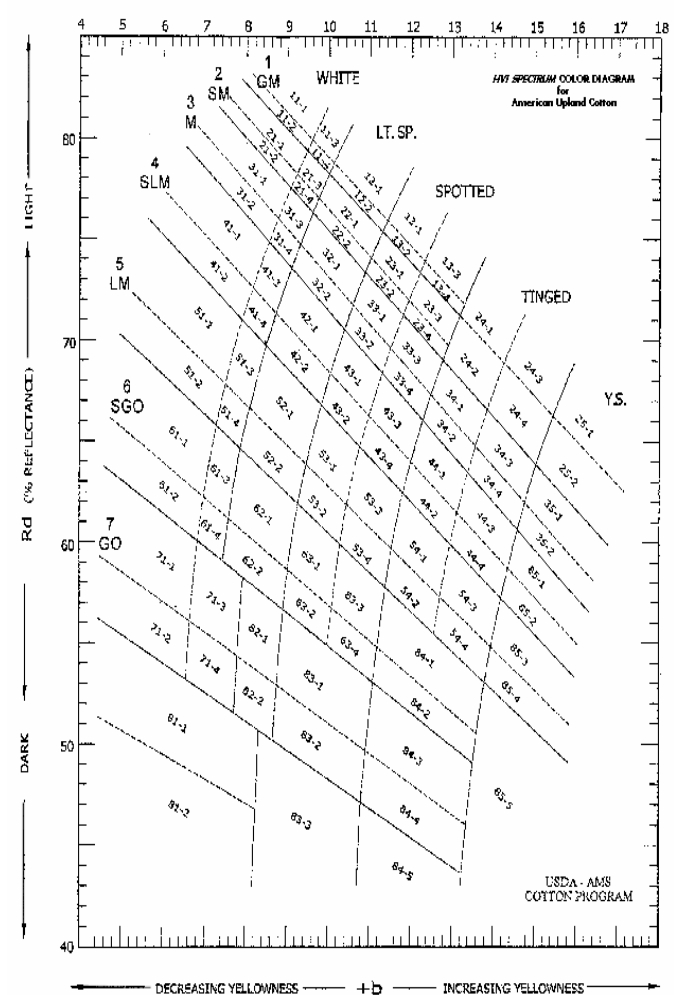

Fig. 2 Nickerson-Hunter colorimeter diagram

\section{Responsibility of Spinner}

If barré problem on fabric is caused by fiber preparation (mixing) or yarn manufacturing stage then it's not possible to take any preventive action to optimize it because a certain amount of yarn is all ready produced from a specific lot of raw cotton. Generally this barré problem may arise after some month even after a year. Because, firstly the yarn is purchased by any knitting mill, then it produces knit-fabric from this yarn on a suitable time. Its time suitability may be depends on its order collection, shipment date, machine availability, requirements of further processing stage, over all buyers demand etc. That's why it is not impossible to make storage of yarn for many months even for a year by the store department of knitting mills. But it is quite possible to make a claim on quality requirement of yarn even after one year from the yarn purchasing date. There is no way to get a prediction about barré problem in yarn manufacturing stage. Spinning mills always stay on risk at facing barré problem from the further processing zone; as like dyeing or finishing section.

\section{Action to be Taken}

Therefore, to avoid this quality concern problem, quality department of spinning mills has to take initiative from the beginning stage of yarn manufacturing as like raw cotton selection. High volume Instrument (HVI) offered by Uster is used in our most of the export oriented mills. By evaluating the raw cotton test result delivered from HVI machine, mixing is planned in such a way that it is possible to avoid the problem.

As like if it is possible to ensure the uniform distribution of average Micronaire of all lay down then it should be assumed that number of fibre in a given cross section of yarn is also distributed uniformly [6]. By which variation of dyeability of yarn is also minimized. On the other hand if a wide range of Micronaire value is present in a specific lot then it is hard to maintain the average Micronaire value in all laydown which results in uneven distribution of fibre in the cross section of yarn. It is mentioned in here that each fibre in the yarn absorbs and fix dye to it. That's why number of fibre in yarn cross section defined how light or deep the shed is. If we want to get a same shed throughout the fabric then it is necessary to exhibit constant number of fibre in yarn cross section. And this is only possible when we ensure the constant average Micronaire for all laydown of a same lot.

In the same way, we have to ensure the representation of homogeneous color grade in each laydown. A lot of cotton, where thousands of bales may remain, has various color grades from GM to BG and White to Yellow stained. If it is possible to select the bale for each laydown from the same lot proportionally on behalf of its color then uniform distribution will be established [7]. Let us consider a lot of CIS raw cotton of approximately 500 tons having 2255 bales. By plotting Mic values of each individual 
Table 1 Average Micronaire 4.420 and Micronaire CV\% 5.76

\begin{tabular}{|c|c|c|c|c|c|c|c|c|c|c|c|c|c|}
\hline Mic & $\begin{array}{c}\text { GM } \\
\text { (White) }\end{array}$ & $\begin{array}{c}\text { GM } \\
\text { Lt.Sp.) }\end{array}$ & $\begin{array}{c}\text { GM } \\
\text { (Sptd.) }\end{array}$ & $\begin{array}{c}\text { SM } \\
\text { (White) }\end{array}$ & $\begin{array}{c}\text { SM } \\
\text { (Lt.Sp.) }\end{array}$ & $\begin{array}{c}\text { SM } \\
\text { (Sptd.) }\end{array}$ & $\begin{array}{c}\text { M } \\
\text { (White) }\end{array}$ & $\begin{array}{c}\text { M } \\
\text { (Lt.Sp.) }\end{array}$ & $\begin{array}{c}\text { M } \\
\text { (Sptd.) }\end{array}$ & $\begin{array}{c}\text { SLM } \\
\text { (White) }\end{array}$ & $\begin{array}{c}\text { SLM } \\
\text { (Lt.Sp.) }\end{array}$ & $\begin{array}{c}\text { LM } \\
\text { (Lt.Sp.) }\end{array}$ & Total \\
\hline $\mathbf{3 . 0}$ & & & & 1 & & & & & & & & & $\mathbf{1}$ \\
\hline $\mathbf{3 . 6}$ & & & 1 & & & & & & & & & & $\mathbf{1}$ \\
\hline $\mathbf{3 . 7}$ & 2 & & & & & & & & & & & & $\mathbf{2}$ \\
\hline $\mathbf{3 . 8}$ & & & & & 1 & & & & & & & & $\mathbf{1}$ \\
\hline $\mathbf{3 . 9}$ & 3 & 4 & & 9 & 1 & 1 & 6 & 2 & & & & & $\mathbf{2 6}$ \\
\hline $\mathbf{4 . 0}$ & 32 & 12 & 4 & 24 & 7 & 1 & 17 & 4 & & 2 & 3 & & $\mathbf{1 0 6}$ \\
\hline $\mathbf{4 . 1}$ & 68 & 44 & 40 & 44 & 14 & 3 & 26 & 6 & 3 & 10 & 1 & 1 & $\mathbf{2 6 0}$ \\
\hline $\mathbf{4 . 2}$ & 82 & 66 & 38 & 54 & 12 & 10 & 22 & 7 & 3 & 4 & 2 & & $\mathbf{3 0 0}$ \\
\hline $\mathbf{4 . 3}$ & 74 & 25 & 17 & 47 & 8 & 2 & 23 & 5 & 2 & 4 & 1 & & $\mathbf{2 0 8}$ \\
\hline $\mathbf{4 . 4}$ & 113 & 12 & 2 & 66 & 8 & & 31 & 10 & & 5 & 4 & & $\mathbf{2 5 1}$ \\
\hline $\mathbf{4 . 5}$ & 173 & 15 & 2 & 104 & 12 & & 32 & 3 & & 2 & 4 & & $\mathbf{3 4 7}$ \\
\hline $\mathbf{4 . 6}$ & 122 & 11 & & 85 & 19 & 1 & 27 & 16 & & 1 & 3 & 1 & $\mathbf{2 8 6}$ \\
\hline $\mathbf{4 . 7}$ & 90 & 11 & 2 & 82 & 18 & & 33 & 2 & 1 & 8 & 4 & 1 & $\mathbf{2 5 2}$ \\
\hline $\mathbf{4 . 8}$ & 42 & 4 & 1 & 47 & 7 & & 17 & 11 & & 7 & 1 & & $\mathbf{1 3 7}$ \\
\hline $\mathbf{4 . 9}$ & 22 & 1 & & 21 & 3 & & 5 & 1 & & 4 & & & $\mathbf{5 7}$ \\
\hline $\mathbf{5 . 0}$ & 5 & & & 6 & & & 4 & & & & & & $\mathbf{1 5}$ \\
\hline $\mathbf{5 . 1}$ & 1 & & & & & & 1 & & & & & & $\mathbf{2}$ \\
\hline $\mathbf{5 . 2}$ & 1 & & & 1 & & & & & & & & & $\mathbf{2}$ \\
\hline $\mathbf{5 . 3}$ & & & & 1 & & & & & & & & & $\mathbf{1}$ \\
\hline Total & $\mathbf{8 3 0}$ & $\mathbf{2 0 5}$ & $\mathbf{1 0 7}$ & $\mathbf{5 9 2}$ & $\mathbf{1 1 0}$ & $\mathbf{1 8}$ & $\mathbf{2 4 4}$ & $\mathbf{6 7}$ & $\mathbf{9}$ & $\mathbf{4 7}$ & $\mathbf{2 3}$ & $\mathbf{3}$ & $\mathbf{2 2 5 5}$ \\
\hline
\end{tabular}

bale of this lot against it's color grade, we found table: 1 . The data shown in table-1 was originally adopted from a HVI cotton test result of 2255 cotton bales [8]. Later the HVI data was converted to a concise format by means of a sorting software. This concise format is shown in table- 1 . The original HVI data which is available to the author is very huge and beyond scope of publication.

It shows in table 1 that some bales are found abnormal in accordance with their Micronaire and color grade. Some bale shows low Micronaire value (3.0-3.9) and some other shows high Micronaire value(5.0-5.3). Some of the bale are categorized as bad color grade as like SM(spotted), M(sp.), SLM(lt. sp.) LM(lt. sp.). Another important aspect is that: the Micronaire value and color grade of some bales are scattered due to their wide range which shoot up the $\mathrm{CV} \%$ of Micronaire value beyond the limit. Therefore, it would be effective for us to consider a specific Micronaire range and also deduct the individual bale of bad color groups. It is assumed that by taking Micronaire range 4.04.9 and discarding the specific bales having dull color as like $\mathrm{SM}$ (sptt.), $\mathrm{M}$ (sp.), SLM(lt.sp) to LM (lt.sp) we able to form a polarized shape of above format which leads to lower $\mathrm{CV} \%$ of Micronaire value. And it is shown in Table 2.

Then approximately 100 bales may be deducted which is negligible. These deducted bales may be utilized later for producing any odd lot having lower quality requirement. By segregating these odd bales we enable to reduce cv\% which help us to make a uniform mixing where more consistency should be achieved.

Now we assign bale management system where 2152 bales are considered which is about 475 tons in weight (each CIS bale contain $220 \mathrm{~kg}$ yarn). It is possible to produce approximately 350 ton and 400 ton yarns from this lot for comb and card process respectively. A medium capacity spinning mill having production volume of 15 MT have to continue this same lot for approx. one month.

\subsection{Constant Process Parameter}

Before introducing the lot in production we also have to make a spin plan which is subjected through the whole month. We must have to fix up waste segregation $\%$ at the initial stage of production whether it is 7 to $15 \%$ for card process and 25 to $30 \%$ in combed process. 
Table 2 Average Micronaire 4.424 and Micronaire CV\% 5.42

\begin{tabular}{|r|r|r|r|r|r|r|r|r|r|}
\hline Mic & $\begin{array}{r}\text { GM } \\
\text { (White) }\end{array}$ & $\begin{array}{r}\text { GM } \\
\text { (Lt.Sp.) }\end{array}$ & $\begin{array}{r}\text { GM } \\
\text { (Sptd.) }\end{array}$ & $\begin{array}{r}\text { SM } \\
\text { (White) }\end{array}$ & $\begin{array}{r}\text { SM } \\
\text { (Lt.Sp.) }\end{array}$ & $\begin{array}{r}\mathbf{M} \\
\text { (White) }\end{array}$ & $\begin{array}{r}\mathbf{M} \\
\text { (Lt.Sp.) }\end{array}$ & $\begin{array}{r}\text { SLM } \\
\text { (White) }\end{array}$ & Total \\
\hline $\mathbf{4 . 0}$ & 32 & 12 & 4 & 24 & 7 & 17 & 4 & 2 & $\mathbf{1 0 2}$ \\
\hline $\mathbf{4 . 1}$ & 68 & 44 & 40 & 44 & 14 & 26 & 6 & 10 & $\mathbf{2 5 2}$ \\
\hline $\mathbf{4 . 2}$ & 82 & 66 & 38 & 54 & 12 & 22 & 7 & 4 & $\mathbf{2 8 5}$ \\
\hline $\mathbf{4 . 3}$ & 74 & 25 & 17 & 47 & 8 & 23 & 5 & 4 & $\mathbf{2 0 3}$ \\
\hline $\mathbf{4 . 4}$ & 113 & 12 & 2 & 66 & 8 & 31 & 10 & 5 & $\mathbf{2 4 7}$ \\
\hline $\mathbf{4 . 5}$ & 173 & 15 & 2 & 104 & 12 & 32 & 3 & 2 & $\mathbf{3 4 3}$ \\
\hline $\mathbf{4 . 6}$ & 122 & 11 & & 85 & 19 & 27 & 16 & 1 & $\mathbf{2 8 1}$ \\
\hline $\mathbf{4 . 7}$ & 90 & 11 & 2 & 82 & 18 & 33 & 2 & 8 & $\mathbf{2 4 6}$ \\
\hline $\mathbf{4 . 8}$ & 42 & 4 & 1 & 47 & 7 & 17 & 11 & 7 & $\mathbf{1 3 6}$ \\
\hline $\mathbf{4 . 9}$ & 22 & 1 & & 21 & 3 & 5 & 1 & 4 & $\mathbf{5 7}$ \\
\hline Total & $\mathbf{8 1 8}$ & $\mathbf{2 0 1}$ & $\mathbf{1 0 6}$ & $\mathbf{5 7 4}$ & $\mathbf{1 0 8}$ & $\mathbf{2 3 3}$ & $\mathbf{6 5}$ & $\mathbf{4 7}$ & $\mathbf{2 1 5 2}$ \\
\hline
\end{tabular}

Same fibre lot having same process parameter may generate barré problem due to different waste segregation $\%$ for the two different days. Because waste segregation\% indicates how amount of short and immature fibre present in material.

It is recommended to follow a same process parameter from blow room to simplex for a lot. Process parameter which specially get priority in this regard is like that: beater rpm in $\mathrm{m} / \mathrm{min}$ of blow room, production in $\mathrm{kg} / \mathrm{hr}$ of each carding machine, production speed of each draw frame including constant leveling intensity and leveling action point and constant simplex speed and twist per inch of roving [9].

Yarn of same count produced form same cotton lot should have similar cross sectional diameter with a CV\% of yarn count to be below 1 including uniform twist distribution (TPI) of same direction (eg: Z- twist).To make the package of yarn of same lot, winding on speed should be same and in tolerable range to avoid excessive hairiness. It is also better to penetrate same percentage of moisture regain to the yarn of same lot.

\subsection{Selection of Bales for each laydown}

The most sensitive issue is the bale selection. Bale selection should be done in such a way that it is possible to achieve smooth fibre property variation with in a laydown and across a series of laydowns. It is to be mentioned that, this process is continued for approximate one month. A medium capacity spinning mill has to consume 80 to 90 bales to get production of 15 MT per day. Depends on their floor layout plan, if there is a availability of space under bale plucker machine they may able to accommodate all bales (80 to 90 per day approx.) in a single laydown. Otherwise they easily select two mix-laydowns per day each having 40 to 45 bales and then the number of lay down is above 50 (total bales no: 2152). Now the matter is that: How they select these 40 bales for each lay down where the prominence of Micronaire and color grade is equal.

Now we are focused on table No. 2. There are ten successive Micronaire values (4.0 to 4.9) in our selective range. To assure the uniform distribution of Micronaire value it is necessary to select the bales against their Micronaire value in a proportional way whereas, average Micronaire is constant for each laydown with a limited CV\%. After that, we have to focus on color grade. The bale numbers which are already mentioned to be 40 or 45 for a laydown should be select in such a way so that they not only represent all these Micronaire value in equilibrium proportion but also ensure the uniform distribution of color grade. Actually the job is easily performed by any sorting software.

If we able to distribute the bales in each lay down homogenously in regard to their Micronaire and color grade then it is possible to minimize the variation of number of fibre in yarn cross section. Each individual fibre of yarn cross section absorbs dyes and finishes. So, uniform number of fibre in yarn cross section having homogenous Micronaire and color grade expose the required shade after dyeing without variation.

By performing this operation from initial stage, we able to minimized fabric barré problem. 


\section{Conclusion}

Barré problem arises in fabric due to fluctuation of number of fibres in yarn cross section. And the Micronaire value of cotton determines the no. of fibre in yarn cross section. By performing the above procedure for any cotton lot, it is possible to maintain the average Micronaire value with a limited $\mathrm{CV} \%$ and also possible to distribute the color grades uniformly against it's Micronaire value. So we can say unanimously that, the above procedure of bale selection for each lay down confirms the uniform distribution of fibre in yarn cross section which leads to minimize of fabric Barré problem.

\section{References}

[1] Yoseph M. Yankey, "The solution for controlling fabric barré” 1996 Beltwide cotton conference, USA

[2] Textile Terms and Definitions (tenth Edition), A Textile Institute Publication, P 20.

[3] Cotton Incorporated "Barré”, Technical Bulletin, 1992 Published by USDA.
[4] Cotton division, USDA "The Classification of Cotton” Agricultural Handbook 566

[5] Operating Manual "HVI Spectrum" published by USTER, Switzerland.

[6] J. E. Booth, "Principle of Textile Testing”. P167.

[7] Gabriele Peters, "Application of AFIS maturity measurement in cotton yarn manufacturing." Textile Month Oct'1998

[8] Above mentioned cotton lot is imported by Asia Composite Mills, Gazipur and test was conducted in their Q.C lab under Author's supervision in 2007.

[9] Herbet T. Pratt, "Some causes of Barre", Knitting Times, June 13, 1977 P18-22.

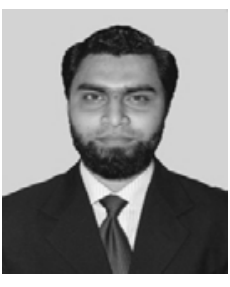

Md. Sanuwar Rashid is working as a lecturer in Textile engineering department of Daffodil International University. $\mathrm{He}$ obtained his B.Sc. degree in Textile Technology from College of Textile Technology under University Of Dhaka in 2004. He has two years work experience on cotton spinning technology. 